\title{
INTEGRAÇÃO DO PROGRAMA SAÚDE NA ESCOLA POR MEIO DE AÇÕES DE PROMOÇÃO E PREVENÇÃO DURANTE O ESTÁGIO CURRICULAR SUPERVISIONADO DE ENFERMAGEM: relato de experiência
}

\author{
Elton Júnior Ferreira ROCHA ${ }^{1}$ \\ Hilda Maria Cardoso PEIXOTO ${ }^{1}$ \\ Valéria Cristina LOPES ${ }^{1}$ \\ Valquíria Ferreira SILVA ${ }^{1}$ \\ Carolina Reis ALVES ${ }^{2}$
}

Patrick Leonardo Nogueira da SILVA ${ }^{3}$

\begin{abstract}
${ }^{1}$ Acadêmico(a) de Enfermagem, Faculdades Unidas do Norte de Minas/FUNORTE. Montes Claros (MG), Brasil. E-mail: juniornuber8@hotmail.com; hildapeixoto03@hotmail.com; leiteferreirajunior@hotmail.com; kirah_fernandes@hotmail.com

${ }^{2}$ Enfermeira, Especialista em Saúde da Família, Mestre e Doutoranda em Ciências da Saúde, Professora do curso de Enfermagem das Faculdades Unidas do Norte de Minas/FUNORTE. Montes Claros (MG), Brasil. E-mail: carolina.calreis@yahoo.com.br

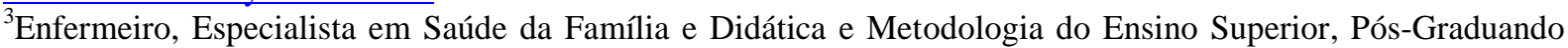
em Enfermagem do Trabalho, Faculdade de Guanambi/FG. Guanambi (BA), Brasil. E-mail: patrick_mocesp70@ hotmail.com
\end{abstract}

Recebido em: 12/11/2015 - Aprovado em: 14/07/2016 - Disponibilizado em: 18/12/2016

\begin{abstract}
RESUMO
Objetivo: relatar a integração do Programa Saúde na Escola por meio de ações de promoção e prevenção durante o estágio curricular supervisionado de enfermagem. Relato de experiência: trata-se de um estudo descritivo, observacional, com suporte em um relato de experiência acadêmica. $\mathrm{O}$ mesmo foi desenvolvido durante o período de março a junho de 2015 com estudantes de instituições públicas de ensino adscrita na área de abrangência de uma Unidade Básica de Saúde na qual faziam parte do Programa Saúde na Escola. Foi realizada educação em saúde sobre diversos temas, também aconteceu vacinação contra o HPV para adolescentes e medição de peso e altura. Nota-se que com a educação em saúde as atividades educativas têm o objetivo de capacitar os escolares para uma vida saudável e segura visando à promoção da saúde na escola, a qual necessita do profissional enfermeiro atuando em espaços diferentes, mas principalmente nos colégios. Considerações finais: o desenvolver dessas ações possibilitou um maior conhecimento sobre a atuação da enfermagem no Programa Saúde na Escola, promovendo uma visão ampla do conceito de saúde diante da interação na realidade da comunidade.

Palavras-chave: Saúde Escolar; Enfermagem; Educação em Saúde.

\section{INTEGRATION OF THE PROGRAM HEALTH IN THE SCHOOL FOR MEANS OF ACTIONS OF PROMOTION AND PREVENTION DURING THE SUPERVISED CURSE STAGE OF NURSING: experience report}

\begin{abstract}
Objective: to report the integration of the School Health Program through promotion and prevention in the curriculum supervised nursing actions. Experience report: This is a descriptive, observational, supported on a report of academic experience. The same was developed during the period from March to June 2015 with students from public institutions of education enrolled in the catchment area of a Basic Health Unit in which were part of the School Health Program. Health education was held on various topics, also it happened HPV
\end{abstract}


vaccination for adolescents and measuring weight and height. Note that with health education educational activities aim to empower school for a healthy and safe life aimed at health promotion at school, which need professional nurse working in different areas, but mainly in schools. Final considerations: the development of these actions enabled a better understanding of the role of nursing in the School Health Program, promoting a broad concept of health vision on interaction in community reality.

Keyword: School Health; Nursing; Health Education.

\section{INTRODUÇÃO}

A escola, que tem como encargo primordial desenvolver métodos de ensinoaprendizagem, exerce papel fundamental na formação e atuação das pessoas em todos os campos da vida social. Juntamente com outros espaços sociais, ela cumpre papel decisivo na formação dos educandos, na percepção e constituição da cidadania. Os profissionais da saúde, por meio do Programa Saúde na Escola (PSE), em conjunto com os educadores, têm o papel de minimizar os riscos existentes para crianças, adolescentes e adultos por meio de ações de saúde (BRASIL, 2009).

A Educação em Saúde envolve conhecimentos e práticas focalizada para prevenção de patologias e promoção da saúde, com suporte em saberes científico, intermediado pelo profissional de saúde, e em saber popular, favorecendo autonomia dos indivíduos envolvidos, o que os torna sujeitos ativos e transformadores de sua própria vida e/ou do seu meio social. A prática de Educação em Saúde deve ser exercida por todos profissionais que compõem a equipe de Saúde da Família, por meio da assistência integral e contínua às famílias da área adscrita, enfrentando os determinantes do processo saúde-doença e incentivando o autocuidado (ALVES, 2005).

O trajeto histórico das práticas de Educação em Saúde no Brasil teve início no final do século XIX, em meio ao surgimento de epidemias que causavam transtornos à economia. As práticas de educação estavam centralizadas às classes subalternas e tinham como característica o autoritarismo, impondo medidas de saneamento e urbanização, sem considerar o contexto político-social e as condições de vida e de trabalho na saúde. As mudanças aconteceram na década de 1940, quando a culpabilização dos problemas de saúde deixa de ser pessoal e passa a ser coletiva (ALVES, 2005).

O método educativo deve ser coletivo, de forma ampla, articulado ao sistema de saúde, levando-se em conta a singularidade local por meio da contextualização do problema, tornando-se imprescindível a presença do diálogo entre os atores envolvidos, contribuindo para transformar a realidade em direção à integralidade (BESEN et al., 2007).

A Educação em Saúde pode ser praticada entre família, na escola, no 
trabalho ou em qualquer lugar comunitário; contudo, os mais comuns à prática de educação voltada para criança e adolescentes são a escola e o ambiente familiar. Cabe ao colégio contribuir para o desenvolvimento integral de estudantes no seu período escolar, proporcionando momentos favoráveis à sua aprendizagem, além de promover o desenvolvimento da sua educação na sociedade. À família, cabe dar continuidade a este processo educativo, visando, sobretudo, à saúde dos seus filhos, que está intimamente ligada ao ambiente físico e emocional da escola, do lar e da comunidade. $O$ trabalho em saúde não deve ser repassado de maneira abstrata, distante da realidade, e sim com práticas vivenciadas pelas crianças (BARBA; MARTINEZ; CARRASCO, 2003).

Esta experiência relatada pode ser classificada como Educação e Saúde na Escola, em função da proposta de se realizarem atividades com temas considerados promotores de saúde, se trabalhados no enfoque da promoção e prevenção. O tema foi referido pela escola, de acordo com a necessidade local, com a diretora, e preceptora de estagio. Foram utilizadas dinâmicas discursão sobre gravidez, vacinação apropriada para o público alvo, vacinação de HPV para as adolescentes e discussão sobre gravidez.

Sendo assim, este estudo objetivou relatar a integração do Programa Saúde na
Escola por meio de ações de promoção e prevenção durante $\mathrm{o}$ estágio curricular supervisionado de enfermagem.

\section{RELATO DA EXPERIÊNCIA}

Trata-se de um estudo descritivo, observacional, com suporte em um relato de experiência das atividades vivenciadas e realizadas nas escolas de abrangência da Estratégia Saúde da Família (ESF) Vila Anália, Montes Claros, Minas Gerais (MG) e realizadas por acadêmicos de Enfermagem do $8^{\circ}$ período noturno das Faculdades Unidas do Norte de Minas (FUNORTE) em parceria com as escolas, com o objetivo de avaliar a saúde dos educandos e possibilitar o encaminhamento para o atendimento e acompanhamento para as alterações identificadas. Entre as atividades realizadas encontram-se: campanha vacinal, antropometria (peso e altura), campanhas sobre drogas, campanhas sobre gravidez na adolescência e alimentação saudável Ainda, são desenvolvidas baseadas no componente II do PSE, baseado na promoção da saúde e prevenção de agravos, que visam oportunizar aos educandos a fazer escolhas mais favoráveis à saúde e de serem protagonistas do processo de produção da própria saúde (BRASIL, 2013). 
Foram realizados encontros e reuniões com a diretoria das escolas para juntos encontrarem e discutirem e programar as ações a serem repassadas para os estudantes, iniciados em março de 2015 e finalizadas em junho do mesmo, mas terá continuidade pela enfermeira da estratégia, com escolares do maternal ao ensino médio diversificando os meios de ensinamentos para cada público. Sendo que, para a abordagem dos temas são utilizadas dinâmicas para promover o envolvimento e protagonismo dos educandos envolvidos.

A equipe de saúde é capacitada para a realização de atividades educativas em saúde com abordagem compatível com os temas de interesse de crianças e adolescentes. Essa capacitação tem vários sentidos: prepara estes profissionais para o estabelecimento de uma relação pedagógica e de confiança entre eles e os alunos da escola, e permite que estes profissionais influam positivamente no aprendizado destes alunos sobre diversos temas ligados à saúde. Além disso, o cronograma das atividades e os temas abordados foram entregues aos professores para que os mesmos trabalhem no decorrer do ano letivo com estes temas em forma de atividades.

Em Março foi realizada pelos acadêmicos do $8^{\circ}$ período, juntamente com a enfermeira e preceptora, a primeira ação de saúde na escola realizada para adolescentes da Escola Municipal Neide Franco Melo sobre gravidez na adolescência. Inicialmente, foi discutido sobre o tema, apresentados os métodos contraceptivos e explicados que eles só iriam evitar uma gravidez indesejada, mas não uma doença sexualmente transmissível. O melhor método para evitar gravidez é o sexo seguro com uso de preservativo feminino ou masculino. Foram abrangidas também as mudanças corporais que acontecem no corpo do adolescente e no corpo da adolescente grávida e riscos existentes para vida da gestante adolescente e para o futuro filho, em debate sobre o tema e esclarecido as principais duvidas da gravidez nesta faixa etária (DINIZ, 2010; SILVA et al., 2012).

O Papiloma vírus Humano (HPV) é um vírus que infecta a pele e mucosas e pode causar câncer do colo de útero e verrugas genitais (BRASIL, 2015). Em março de 2015 também foi realizada vacinação contra o vírus HPV, tendo como público-alvo as adolescentes do sexo feminino entre nove a 11 anos, os pais previamente foram avisados por meio de bilhetes enviados pela coordenação da Escola Municipal Neide Franco Melo e solicitado que as meninas levassem o cartão de vacina ou caderneta da adolescente, a maioria das garotas levou, mas ficaram com medo e dificultaram o 


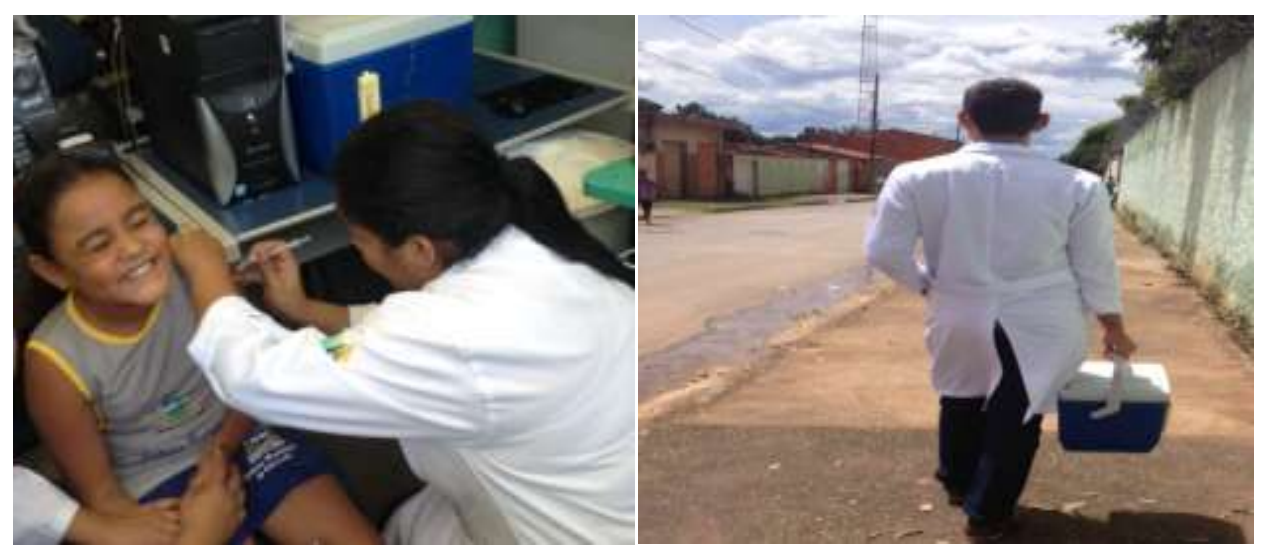

Figura 1 - Vacinação contra o HPV na E. M. Neide Franco Melo.

Neste mesmo mês, especificamente no dia 17 e 18, ainda foram realizadas atividades antropométricas com os alunos das Escolas CEMEI São Marcos e Escola Municipal Neide Franco Melo, foram realizados cálculos de relação peso e altura dos alunos, em seguida jogados no gráfico adequado para cada faixa etária e após os resultados dos cálculos foram marcadas as consultas de enfermagem e consultas médicas para as crianças que se encontraram com peso inadequado para idade (Figura 2).
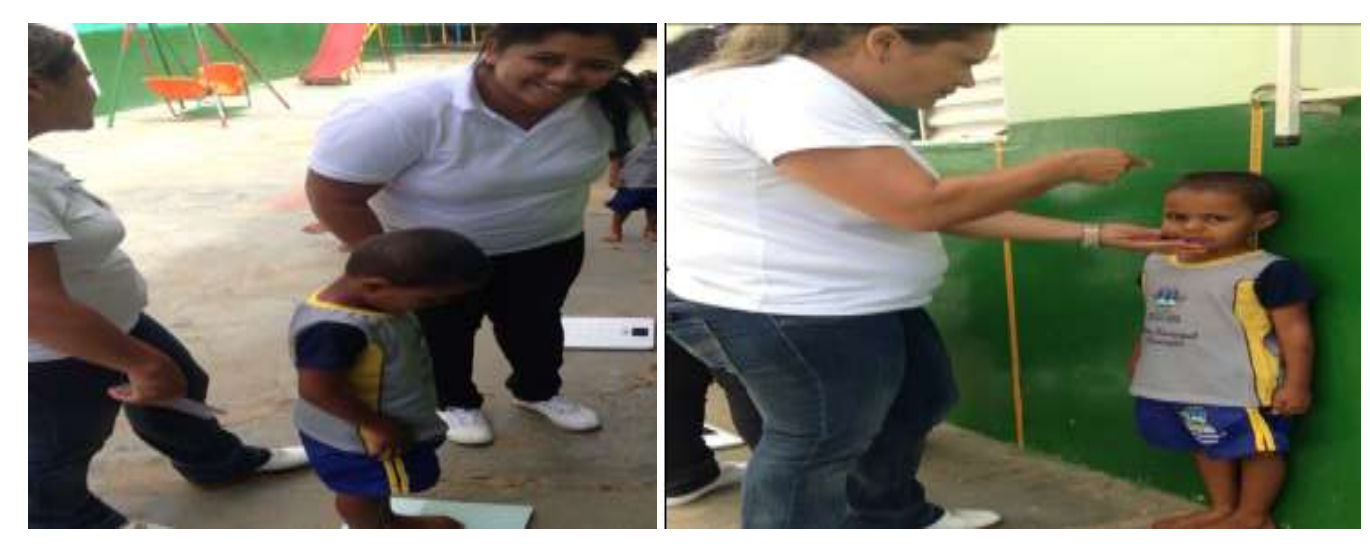

Figura 2 - Antropometria no CEMEI São Marcos e E. M. Neide Franco Melo.

Em Abril, os acadêmicos de responsável técnica da Unidade Básica de enfermagem no internato do $8^{\circ}$ período realizaram um curso online fornecido pelo Ministério da Saúde (MS) e posteriormente uma capacitação ministrado pela Saúde (UBS) sobre NUTRISUS, com o intuito de que tornassem aptos para o manuseio do programa. Em seguida, juntamente com a diretoria da escola, foi 
organizada uma reunião explicativa para os pais das crianças que estudam no CEMEI São Marcos sobre o NUTRISUS. Foram elucidados todos componentes que integram o programa, o objetivo de sua criação, explicado as vantagens, a maneira de introduzi-lo na alimentação e as contraindicações do uso desse suplemento (BRASIL, 2014).

Os pais que aceitaram que os filhos participassem do programa preencheram um formulário de autorização. Foi sugerido pela enfermeira da UBS que todas as crianças que fossem participar do programa, juntamente com os demais familiares, tomassem vermífugo, prescrito pela médica. Foi realizado treinamento com cozinheiras da escola explicando como deveriam ser colocadas nas refeições das crianças e, em seguida, iniciado a adesão ao NUTRISUS. As crianças foram monitoradas e infelizmente, apesar do sache não ter sabor e ser colocado estrategicamente na primeira colher como instruído pelo $\mathrm{MS}$, as crianças não adequaram ao programa, pois as mesmas não estavam se alimentando na escola e relatavam que tinha algo diferente na refeição (Figura 3).

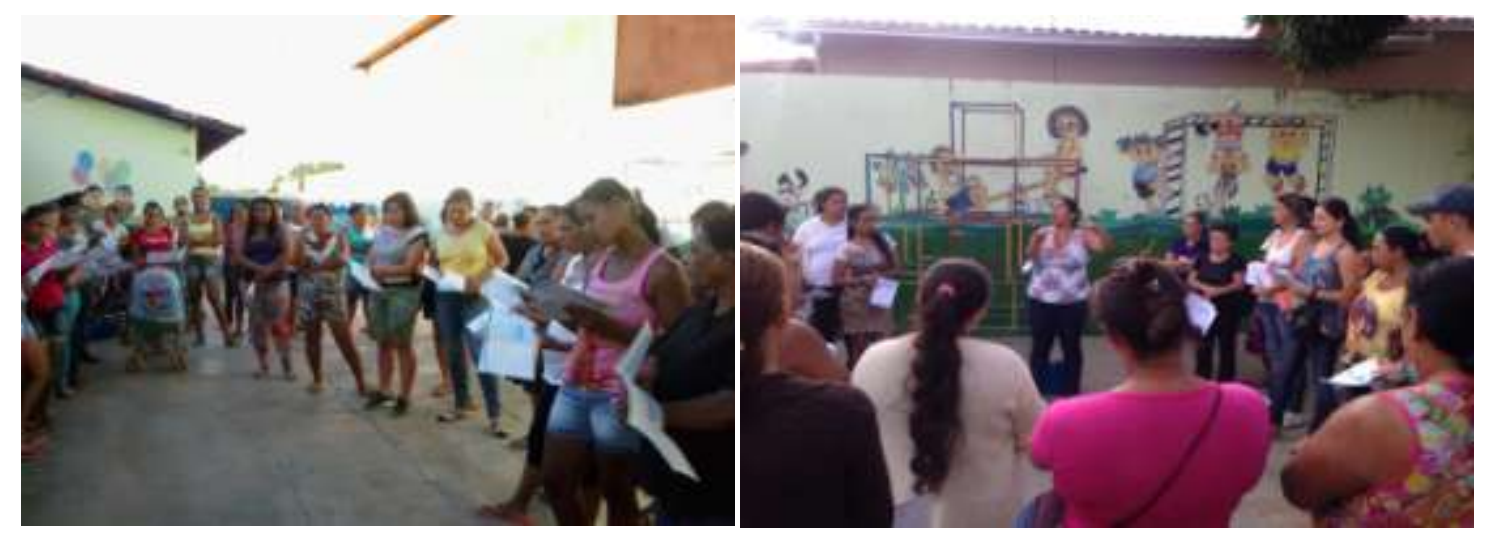

Figura 3 - Capacitação dos pais acerca do NUTRISUS.

A adolescência é uma fase do desenvolvimento humano em que ocorrem muitas mudanças. É uma fase conflituosa da vida devido às transformações físicas e emocionais vividas. Surgem à curiosidade, os questionamentos, a vontade de conhecer, de experimentar o novo, mesmo sabendo dos riscos. É o momento que a adolescente procura sua própria identidade, não mais se fundamentado nas orientações dos pais, mas também nas relações que constrói principalmente com o grupo de amigos (CANAVEZ; ALVES; CANAVEZ, 2010; SILVA et al., 2012).

Em junho de 2015 foi realizada uma campanha contra o uso de drogas 
licitas e ilícitas na Escola Municipal Neide Franco Melo utilizando como recursos fotos ilustrativas, computador, para explicar sobre as drogas e esclarecer dúvidas já existentes nos adolescentes e principalmente demonstrar os danos à saúde causada por estes agentes tóxicos e nocivos e que esta escolha errada poderá trazer consequências para toda a vida.

\section{DISCUSSÃO}

As atividades educativas têm $\mathrm{o}$ objetivo de capacitar os escolares para uma vida saudável e segura visando à promoção da saúde na escola, a qual necessita do profissional enfermeiro atuando em espaços diferentes, mas principalmente em escolas, pois cabem a estas uma função social e política voltada para a transformação da sociedade escolar, relacionando o exercício da cidadania, e aprendizagem, e sendo de considerável relevância o apoio de outros profissionais da saúde, formando atividades multidisciplinares e de forma interdisciplinar (MACIEL et al., 2010).

Além do espaço escolar, as ações podem e devem ser desenvolvidas em nível familiar, com o objetivo de seguimento da educação. Esses elementos são relevantes, pois existem evidências de que o espaço, em conjunto com as condições materiais de vida e o acesso ao serviço de saúde e educação, determina padrões característicos de saúde e patologias das crianças e adolescentes (CASTRO et al., 2005).

A troca de idéias da prática realizada na escola foi realizada com materiais de fácil aquisição e acessíveis, na rotina do trabalho da ESF em estudo, assim como em qualquer outra, tornando possível a realização das atividades e descartando a idéia de que sejam necessários elevados recursos e/ou materiais sofisticados (MOURA; SOUSA, 2002).

A ferramenta de maior potencial para desenvolver a atividade seguiu a linha da criatividade, propiciando o envolvimento com o público-alvo, sendo este um fator determinante para o sucesso do trabalho proposto. O sucesso foi identificado pelos seguintes aspectos: a conquista da integração entre os envolvidos; a aceitação do grupo de crianças, adolescentes, funcionários $\mathrm{da}$ escola e satisfação dos funcionários da Unidade; o diálogo informal por meio do próprio arranjo de organização, em que todos abraçaram a causa para realização destes trabalhos; a ligação que os acadêmicos tiveram com serviço de saúde, na qual se estabeleceu tanto a disposição quanto o desejo de ambos para a continuidade de ações educativas (MACIEL et al., 2010). 
Observou-se que havia interesse, por parte dos alunos, nos assuntos abordados, e os mesmos interagiam conosco fazendo questionamentos. Além disso, as próprias apresentações que realizávamos em forma de teatro, debate e aula ilustrativa, utilizando data-show e com figuras ilustrativas acrescentadas nos slides, proporcionando aprendizado e entretenimento ao mesmo tempo.

\section{CONSIDERAÇÕES FINAIS}

O desenvolver dessas ações possibilitou um maior conhecimento sobre a atuação da enfermagem no PSE, promovendo uma visão ampla do conceito de saúde diante da interação na realidade da comunidade.

Além disso, foi por meio dessas experiências na vida acadêmica que se compreendeu a importância desses trabalhos educativos realizados com crianças e adolescentes. Diante do exposto, infere-se que, as ações de Educação em Saúde estimulam a prevenção de doenças, a promoção da saúde e o engajamento e participação da população nos assuntos correlatos e é por meio dessas ações educativas que se promove a melhoria da qualidade de vida evitando problemas na vida futura dessa população.

A enfermagem tem papel importante nas escolas por se tratar de profissionais que têm capacidade para levantar dados, detectar diagnósticos, planejar e implementar soluções resolutivas, e também por ser mediador de ações educativas para a saúde.

Sugere-se que outros estudos sejam feitos com o intuito de detalhar a Educação em Saúde nas Escolas na Atenção Primária à Saúde (APS).

\section{REFERÊNCIAS}

ALVES, V. S. Um modelo de educação em saúde para o Programa Saúde da Família: pela integralidade da atenção e reorientação do modelo assistencial.

Interface - Comunicação, Saúde e

Educação. Botucatu, v. 9, n. 16, p. 39-52, 2005. Disponível em:

http://www.scielo.br/pdf/icse/v9n16/v9n16 a04.pdf

BARBA, P. C. S. D. et al. Promoção da saúde e educação infantil: caminhos para o desenvolvimento. Paidéia (Ribeirão Preto). Ribeirão Preto, v. 13, n. 26, p. 141-146, 2003. Disponível em: http://www.scielo.br/pdf/paideia/v13n26/0 2.pdf

BESEN, C. B. et al. A estratégia saúde da família como objeto de educação em saúde. Saúde \& Sociedade. São Paulo, v. 16, n. 1, p. 57-68, 2007. Disponível em: http://www.scielo.br/pdf/sausoc/v16n1/06. pdf

BRASIL. Ministério da Saúde. Secretaria de Atenção à Saúde. Departamento de Atenção Básica. Cadernos de Atenção Básica - Saúde na Escola. Brasília, 2009. 96p. Disponível em: http://dab.saude.gov.br/docs/publicacoes/c adernos_ab/abcad24.pdf 
BRASIL. Ministério da Saúde. Ministério da Educação. Passo a passo - adesão semana saúde na escola. Brasília, 2013. Disponível em:

http://portal.mec.gov.br/index.php?option= com_docman\&view=download \&alias $=126$ 47-passo-a-passo-adesao-ssepdf\&category_slug=marco-2013pdf $\&$ Itemid $=30192$

BRASIL. Ministério da Saúde. Secretaria de Atenção à Saúde. Departamento de Atenção Básica. Guia alimentar para população brasileira. $2^{\mathrm{a}} \mathrm{ed}$. Brasília, 2014. Disponível em:

http://189.28.128.100/dab/docs/portaldab/p ublicacoes/guia_alimentar_populacao_bras ileira.pdf

BRASIL. Ministério da Saúde. Secretaria de Vigilância em Saúde. Departamento de Vigilância de Doenças Transmissíveis.

Coordenação Geral do Programa Nacional de Imunizações. Informe técnico da vacina papilomavírus humano $6,11,16 \mathrm{e}$ 18 (recombinante). Brasília, 2015.

Disponível em:

http://www.cosemsrs.org.br/imagens/event os/cli_c1d7.pdf

CANAVEZ, M. F.; ALVES, A. R.; CANAVEZ, L. S. Fatores predisponentes para o uso precoce de drogas por adolescentes. Cadernos UniFOA. Volta Redonda, n. 14, p. 57-63, 2010. Disponível em:

http://web.unifoa.edu.br/cadernos/edicao/1 4/57.pdf

CASTRO, T. G. et al. Caracterização do consumo alimentar, ambiente socioeconômico e estado nutricional de pré-escolares de creches municipais.

Revista de Nutrição. Campinas, v. 18, n. 3, p. 321-330, 2005. Disponível em: http://www.scielo.br/pdf/rn/v18n3/a04v18 $\underline{\text { n3.pdf }}$

DINIZ, N. C. Gravidez na adolescência: um desafio social [Monografia].
Universidade Federal de Minas Gerais, Campos Gerais, 2010. Disponível em: https://www.nescon.medicina.ufmg.br/bibl ioteca/imagem/2336.pdf

MACIEL, E. L. N. et al. Projeto Aprendendo Saúde na Escola: a experiência de repercussões positivas na qualidade de vida e determinantes da saúde de membros de uma comunidade escolar em Vitória, Espírito Santo. Ciência \& Saúde Coletiva. Rio de Janeiro, vol. 15, n. 2, p. 389-396, 2010. Disponível em: http://www.scielo.br/pdf/csc/v15n2/v15n2a 14.pdf

MOURA, E. R. F.; SOUSA, R. A. Educação em saúde reprodutiva: proposta ou realidade do programa saúde da família? Cadernos de Saúde Pública. Rio de Janeiro, v. 18, n. 6, p. 1809-1811, 2002. Disponível em:

http://www.scielosp.org/pdf/csp/v18n6/132 $\underline{\text { 80.pdf }}$

SILVA, P. L. N. et al. Educação sexual: percepções de adolescentes de um prójovem do município de Espinosa/MG através de um relato de experiência. Lecturas Educación Física y Deportes (Buenos Aires). Buenos Aires, v. 17, n. 175, p. 1-7, 2012. Disponível em: http://www.efdeportes.com/efd175/educac ao-sexual-percepcoes-de-adolescentes.htm 